\title{
Entre a biomedicina, a saúde pública e os direitos: um estudo sobre os argumentos do Consórcio Internacional sobre Contracepção de Emergência para promover o acesso aos contraceptivos de emergência em "países em desenvolvimento"*
}

\author{
Luiza Lena Bastos** \\ Miriam Ventura*** \\ Elaine Reis Brandão****
}

\section{Resumo}

Esta é uma pesquisa socioantropológica documental sobre os argumentos do Consórcio Internacional sobre Contracepção de Emergência (ICEC) para ampliar o acesso das mulheres ao método nos "países em desenvolvimento". Três abordagens teóricas foram identificadas: a sanitária, a farmacêutica e a dos direitos. Este trabalho permite refletir sobre os múltiplos sentidos conferidos pelo consórcio aos contraceptivos de emergência como dispositivos biomédicos e sanitários cujo acesso deve se constituir como um direito para as mulheres, pois possibilitam o exercício da autonomia reprodutiva.

Palavras-chave: Contracepção de Emergência, Sexualidade, Gênero, Direitos Reprodutivos, Saúde Reprodutiva.

"Recebido em 07 de dezembro de 2015, aceito em 03 de julho de 2018.

*"Doutoranda em Saúde Coletiva do Instituto de Estudos em Saúde Coletiva, Universidade Federal do Rio de Janeiro, Rio de Janeiro, RJ, Brasil. luizalenab@gmail.com / orcid.org/0000-0002-9206-6773

**** Professora Adjunta do Instituto de Estudos em Saúde Coletiva, Universidade Federal do Rio de Janeiro, Rio de Janeiro, RJ, Brasil. miriam.ventura@iesc.ufri.br / orcid.org/0000-0001-8520-8844

***** Professora Associada do Instituto de Estudos em Saúde Coletiva, Universidade Federal do Rio de Janeiro, Rio de Janeiro, RJ, Brasil. brandao@iesc.ufri.br / http://orcid.org/0000-0002-3682-6985 
Between Biomedicine, Public Health and Rights: A Study About The International Consortium for Emergency Contraception's Arguments to Promote Access to Emergency Contraception in "Developing Countries".

\begin{abstract}
This is a documental and socio-anthropological research about the International Consortium for Emergency Contraception's arguments (ICEC) to expand the Emergency Contraception (EC) access to women in "developing countries". Three theoretical perspectives have been identified: the sanitary, the pharmaceutical and the one about the rights. This work allows us to reflect on Consortium's meanings for ECs, like biomedical and sanitary devices that must be constituted as a right to women as they enable the reproductive autonomy exercise.
\end{abstract}

Keywords: Emergency Contraception, Sexuality, Gender, Reproductive Rights, Reproductive Health. 


\section{Introdução}

Desde a década de 1920, com os estudos de A.S. Parkes e $\mathrm{W}$. Bellerby que perceberam que a ingestão de altas doses de estradiol poderia interferir na gravidez de mamíferos, os contraceptivos de emergência (CE) têm se desenvolvido em meio a disputas morais, econômicas e políticas, que vão repercutir no direito das usuárias ao acesso à tecnologia. Existem hoje quatro "gerações" de CE que assumem a forma farmacêutica de comprimido: o Dietilbestrol; os contraceptivos de pílulas combinadas - pílulas contraceptivas orais (PCO) que contêm estrogênio e progestina, também conhecidos como "método Yuzpe"; o levonorgestrel e as antiprogestinas. Há também outro que se apresenta na forma de um dispositivo intra-uterino (DIU) de cobre (Foster; Wynn, 2012; Prescott 2011; Ellertson 1996; Haspels; Andriesse, 1973; Trussell, 2012).

O Consórcio Internacional sobre Contracepção de Emergência (ICEC) se constitui como uma rede integrada por atores - principalmente organizações filantrópicas, de planejamento familiar e laboratórios farmacêuticos - que têm por objetivo expandir ao redor do mundo o uso dos $\mathrm{CE}$, os quais podem ser utilizados pelas mulheres após as relações sexuais desprotegidas como um recurso importante para evitar a gravidez. O ICEC é o principal interlocutor na discussão e na difusão desse tipo de contraceptivo, tendo sua atuação direcionada para os "países em desenvolvimento".

A existência de um consórcio dedicado exclusivamente a advogar em prol de um tipo específico de contraceptivo materializa a tensão dialética permanente que transpassa a sexualidade, o corpo, o prazer e a reprodução; e nos remete para a discussão da biopolítica e do gerenciamento moral, social $e$ político dos corpos, especialmente do corpo feminino, nos âmbitos da Saúde Pública, da Medicina e do Direito. Considerando a posição estratégica do ICEC como uma organização internacional privilegiada no diálogo com as instâncias das Nações Unidas, Estados e suas agências regulatórias, organizações feministas e de 
saúde, e indústrias farmacêuticas, a investigação buscou identificar deslocamentos nos argumentos do consórcio sobre a ampliação de autonomia e fortalecimento de dispositivos de poder em prol do gerenciamento do corpo feminino.

Para análise do conjunto de argumentos utilizados pelo ICEC em prol da difusão dos CE ao redor do mundo, realizou-se pesquisa socioantropológica, de caráter/natureza documental no conteúdo veiculado no website e na lista de e-mails do consórcio, aberta aos participantes interessados pelo tema. Discute-se como esses atores se mobilizam e discursam sobre a importância desse tipo de contraceptivo. Este estudo foi motivado pela necessidade de ampliarmos no Brasil a discussão sobre o uso dos medicamentos de contracepção de emergência como mais uma tecnologia que possa vir a possibilitar uma melhor vivência da sexualidade e reprodução para mulheres.

Primeiro, apresentamos o Consórcio Internacional sobre Contracepção de Emergência e, em seguida, o percurso metodológico utilizado. Na classificação dos argumentos adotados pelo consórcio, três enfoques foram identificados. O da saúde pública considera que o acesso ao contraceptivo é importante devido aos riscos de uma "gravidez não planejada" ou "não desejada" para a saúde das mulheres, especialmente as adolescentes, e suas implicações relacionadas ao abortamento inseguro. $\mathrm{O}$ enfoque farmacêutico enfatiza segurança, eficácia $e$ mecanismo de ação das pílulas, ressaltando a contracepção de emergência na forma farmacêutica de comprimido, que pode ser utilizado livremente pelas mulheres para evitar as gravidezes imprevistas, destacando-se o fato de não ser abortiva. Por fim, o enfoque dos direitos, em especial os direitos das mulheres $e$ adolescentes ao controle de sua fecundidade $e$ ao acesso aos contraceptivos, incluindo os de emergência, como meios para garantia desses direitos reprodutivos, enfatizando-se o direito à saúde reprodutiva. 


\section{O Consórcio Internacional sobre Contracepção de Emergência}

O estudo e o desenvolvimento do potencial contraceptivo de determinados hormônios, bem como a introdução deles em diversos países, abriu espaço para a articulação de alianças. A discussão sobre a formação das alianças que fundam o Consórcio Internacional sobre Contracepção de Emergência tem o intuito de assinalar o contexto sociopolítico no qual ele emerge.

No mesmo ano em que acontecia a IV Conferência Mundial sobre as Mulheres, em Pequim, em 1995, a Fundação Rockefeller ${ }^{1}$ convocou uma reunião com vários grupos interessados em promover a contracepção de emergência como uma alternativa para o aborto inseguro, especialmente nos "países em desenvolvimento" (Prescott, 2011:103).

O encontro tinha o objetivo de "estabilizar" os CE - na tentativa de facilitar sua aceitação social, reduzindo os embates sobre seus efeitos sobre a saúde sexual e reprodutiva, a sexualidade e a fertilidade e suas repercussões demográficas, econômicas, políticas. Essa reunião foi realizada em Bellagio, na Itália, com a presença de 24 especialistas de todo o mundo, incluindo o pesquisador brasileiro Dr. Elsimar Coutinho (Manica, 2009), da Universidade Federal da Bahia, que atuou como presidente da conferência. Após essa conferência, foi criada a "Declaração de Consenso sobre a Contracepção de Emergência" (Consensus statement on emergency contraception) (WHO, 1995). Nesse documento, os especialistas declaram que é papel dos governos, agências intergovernamentais $e$ organizações não governamentais assegurar que os $\mathrm{CE}$ sejam inclusos nas políticas de planejamento reprodutivo e nas listas de medicamentos essenciais.

Ao todo, sete organizações estavam envolvidas nessa conferência e, posteriormente, fundaram o consórcio: The

\footnotetext{
1 A Fundação Rockefeller é uma fundação filantrópica historicamente preocupada com questões de planejamento familiar (Clarke, 1998). Mais informações disponíveis em: http://www.rockefellerfoundation.org/about-us.
} 
Concept Foundation, International Planned Parenthood Federation (IPPF), Pacific Institute for Women's Health, Pathfinder International, PATH (Program for Appropriate Technology in Health), Population Council, World Health Organization Special Programme of Research, Development, and Research Training in Human Reproduction (WHO/HRP).

O ICEC tem uma estrutura organizacional peculiar. Agrega entre seus membros o setor privado, representado por laboratórios farmacêuticas e o setor público, fazendo parcerias com governos de diversos países. Além disso, integram o ICEC organizações não governamentais diversas, feministas, de defesa dos direitos reprodutivos, com destaque para as de planejamento familiar, como pode ser observado no quadro a seguir (Quadro 1). 
Quadro 1: Parceiros do ICEC - ano de fundação, país de origem e sede da organização

\begin{tabular}{|l|c|c|c|}
\hline \multicolumn{1}{|c|}{ ONG } & $\begin{array}{c}\text { Ano de } \\
\text { fundação }\end{array}$ & País de origem & Sede atual \\
\hline Population Council & 1952 & EUA & EUA \\
\hline $\begin{array}{l}\text { IPPF - International Planned Parenthood } \\
\text { Federation }\end{array}$ & 1952 & Índia & Inglaterra \\
\hline $\begin{array}{l}\text { IPPF - International Planned Parenthood } \\
\text { Federation/Western Hemisphere Region }\end{array}$ & 1954 & Índia & EUA \\
\hline Pathfinder International & 1957 & EUA & EUA \\
\hline $\begin{array}{l}\text { ARHP - Association of Reproductive } \\
\text { Health Professionals }\end{array}$ & 1963 & EUA & EUA \\
\hline PAI Population Action International & 1965 & EUA & EUA \\
\hline $\begin{array}{l}\text { Planned Parenthood Federation of } \\
\text { America }\end{array}$ & 1966 & EUA & EUA \\
\hline $\begin{array}{l}\text { CONRAD - Contraception Research and } \\
\text { Development }\end{array}$ & 1968 & EUA & EUA \\
\hline FCI - Family Care International & 1968 & EUA & EUA \\
\hline Guttmacher Institute & 1968 & EUA & EUA \\
\hline $\begin{array}{l}\text { Bpas - British Pregnancy Advisory } \\
\text { Service }\end{array}$ & 1968 & Inglaterra & Inglaterra \\
\hline PATH & 1970 & EUA & EUA \\
\hline PSI Population Services International & 1970 & EUA & EUA \\
\hline FHI 360 - Family Health International & 1971 & EUA & EUA \\
\hline $\begin{array}{l}\text { HRP - Special programme of research, } \\
\text { development and research training in } \\
\text { human reproduction. }\end{array}$ & 1972 & $\begin{array}{c}\text { Cooperação } \\
\text { Internacional }\end{array}$ & Suíça \\
\hline CFC - Catholics for Choice & 1973 & EUA & EUA \\
\hline Ipas Health, access, rights & 1973 & EUA & EUA \\
\hline $\begin{array}{l}\text { JSI - John Snow Inc. Research \& } \\
\text { Training for Women's Health }\end{array}$ & 1978 & EUA & EUA \\
\hline DKT international & 1989 & Índia & EUA \\
\hline CRR - Center for Reproductive Rights & 1992 & EUA & EUA \\
\hline Meridian Group International & 1997 & EUA & EUA \\
\hline Ibis Reproductive Health & 2002 & EUA & EUA \\
\hline Gynuity Health Projects & 2009 & EUA & EUA \\
\hline Woman Care Global & EUA & EUA \\
\hline Prosalud Interamericana Foundation & & \\
\hline
\end{tabular}


Cada elemento da estrutura organizacional do ICEC confere apoio financeiro e político para que novos contraceptivos como os $\mathrm{CE}$, possam ser desenvolvidos. O consórcio representa um conjunto organizacional bem sucedido no desenvolvimento $e$ na introdução de um novo contraceptivo, reunindo uma variedade de expertises - desde o conhecimento biomédico até a habilidade de lidar com complexos requerimentos regulatórios e atividades de propaganda - em especial para promover acesso à CE em "países em desenvolvimento".

A constante menção ao "acesso para países em desenvolvimento", no corpus analisado, expressa preocupações na direção de conter o crescimento populacional e a perpetuação da pobreza. Diversos documentos assinalam os CE como parte do "planejamento familiar", pois seria a intervenção mais custoefetiva para "aliviar" a pobreza. Conforme achados recentes (Bastos; Ventura; Brandão, 2017), observa-se o distanciamento de uma perspectiva que busque legitimar o exercício autônomo da sexualidade e da reprodução enquanto dimensões emancipadoras dos sujeitos.

As organizações internacionais e de planejamento familiar que compõem atualmente o ICEC tiveram um grande impacto na regulação da sexualidade e da reprodução não só no Brasil como em toda a América Latina ou nos países "em desenvolvimento". De acordo com Bonan (2005), essas organizações abriram brechas para posicionamentos tanto pró-natalistas, para uma visão maternalista da mulher e contribuíram para ideia de procriação como finalidade maior da sexualidade feminina, quanto antinatalistas com a emergência da preocupação com a "explosão demográfica". O predomínio do enfoque demográfico desses programas num contexto de poucas condições democráticas $e$ transparentes, como foi o contexto da década de 1960 até meados da década de 1980 no Brasil, significou um reforço do poder e da autoridade médica e, em alguns casos, estatal sobre o corpo e a fecundidade feminina.

As grandes ações do consórcio foram motivadas porque almejava o controle populacional a partir de um "back up 
control', ou seja, conferir um reforço contraceptivo caso houvesse "erros" com a administração da pílula hormonal de uso regular.

Em certos contextos ocidentalizados, esse contraceptivo foi bem aceito. Percebe-se que o uso dos CE pode estar associado a certa liberdade sexual e controle feminino da reprodução: a pílula é tomada em até 120 horas em uma única dose e ainda permite certa discrição no momento da ingestão. Embora os laboratórios farmacêuticos aleguem que as pílulas contraceptivas sejam universais, independente de contextos socioculturais específicos, de certa socialização contraceptiva e das relações entre gêneros (Cabral, 2011), elas não se dirigem para uma usuária qualquer, mas para uma mulher disciplinada o bastante para tomar de forma emergencial a "medicação", acostumada a exames ginecológicos e visitas regulares a serviços médicos. Essa perspectiva de enquadre do uso dos contraceptivos revela que eles são tecnologias culturalmente específicas, com variações mesmo dentro de uma cultura (Oudshoorn, 1994). No contexto do oeste africano, por exemplo, a pesquisa realizada por Teixeira et alii (2012) demonstrou que representações sobre CE nem sempre se referem à noção de "emergência" e "backup"; seu uso pode ser regular e não urgente. Além disso, os autores discutem, seguindo Desclaux e Lévy (2003), que as diversas percepções negativas encontradas no estudo, em relação a sua toxicidade e os efeitos adversos, são também expressões políticas. A hipótese levantada é a de que a resistência encontrada pela difusão de contraceptivos hormonais gira em torno de questões de autonomia sexual $e$ reprodutiva.

\section{Metodologia}

Apresentamos abaixo o mapa do website do consórcio (http://www.cecinfo.org), que representa a plataforma virtual investigada neste trabalho. 
Figura 1: Mapa do website do Consórcio Internacional sobre Contracepção de Emergência.

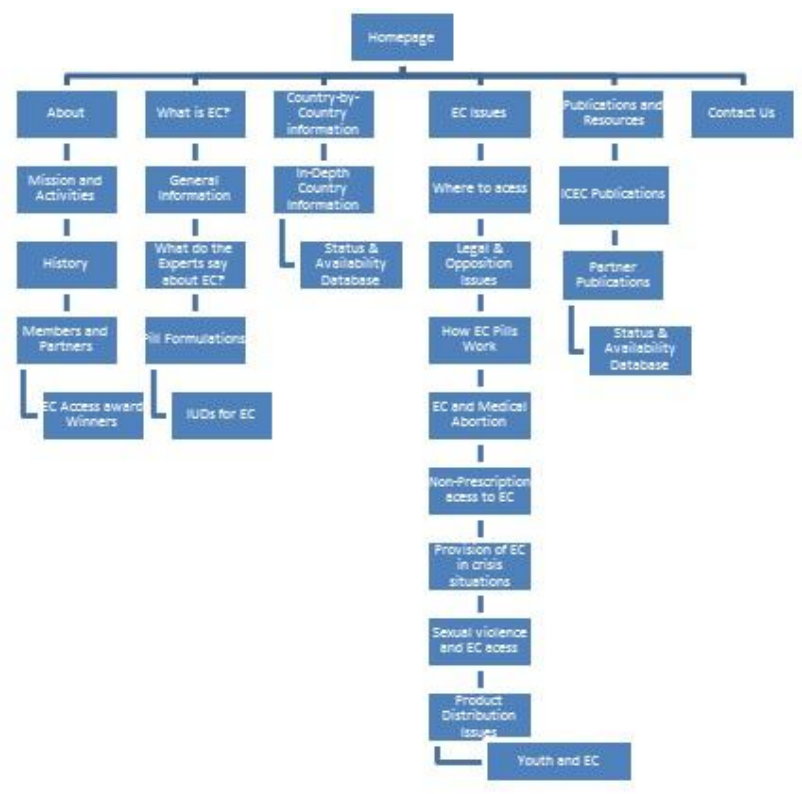

Dentro dessas seções estão contidos os documentos selecionados para análise. As seções "What's EC?" e "EC Issues" não continham documentos anexados, portanto, as próprias seções e suas subseções foram tomadas enquanto documentos.

O primeiro passo da pesquisa foi selecionar os documentos veiculados no website do consórcio para posterior análise. Para tanto, foram selecionados e privilegiados na análise sistemática $e$ em profundidade os documentos da subseção "ICEC Publications" por conterem apenas publicações dos membros do ICEC (comitê diretivo e comitê de apoio técnico). Entretanto, documentos contidos nas outras seções também foram acessados por conterem material importante para a melhor compreensão da organização e da problemática do estudo. Nelas há documentos publicados por 
parceiros do consórcio, os quais dão apoio material e político para o ICEC (Quadro 1). A coleta desse material foi realizada em etapas. Nos meses de agosto e setembro de 2013, foram realizadas visitas "flutuantes" para "captar os documentos" e, em outubro de 2013, visitas em maior profundidade já selecionando o material empírico. Novamente, em 2014, nos meses de março, abril e maio, visitou-se o website em profundidade.

No caso dos documentos, os procedimentos de seleção $e$ inclusão obedeceram aos seguintes passos: 1) leitura flutuante do conjunto de documentos (aproximadamente 133); 2) identificação de categorias temáticas recorrentes nos documentos lidos (gravidez "não planejada", gravidez "não desejada", gravidez na adolescência, saúde pública, segurança, eficácia, mecanismo de ação, venda $\mathrm{OTC}^{2}$, direito ao acesso aos contraceptivos); 3) seleção dos documentos que abordavam prioritariamente os temas identificados como estratégicos para argumentação do consórcio. Foram selecionados 83 documentos para análise em profundidade.

Além disso, resgatamos as discussões de uma lista de e-mails utilizada por membros do consórcio que aceita como participante qualquer pessoa que se interesse pelo tema da $\mathrm{CE}$. A entrada na lista de e-mail ocorreu no dia 5 de abril de 2012 com o objetivo de acompanhar os debates. Até o dia 2 de maio de 2014, foram recebidos 211 e-mails.

Com certo tempo de participação na lista, foi possível perceber que as discussões que ali ocorriam se assemelhavam muito aos elementos que estávamos encontrando no website, por isso decidimos focar atenção nos documentos, mas acompanhando a discussão na lista, o que foi muito importante para a reflexão sobre os argumentos do ICEC. Nessa lista, foram

2 OTC ou Over-the-counter é uma expressão utilizada por agências regulatórias de medicamentos como o Food and Drug Administration (FDA) e a Agência Nacional de Vigilância Sanitária (ANVISA) para denominar medicamentos considerados seguros e eficazes e que sejam isentos de prescrição médica, podendo ser obtidos em gôndolas de farmácias (FDA, s/d.). 
selecionados os e-mails que apresentavam as temáticas já identificadas nos documentos.

Quadro 2: Número de documentos selecionados

\begin{tabular}{|c|c|c|}
\hline \multicolumn{2}{|c|}{ Seção do website } & $\begin{array}{c}\text { Número de documentos } \\
\text { selecionados }\end{array}$ \\
\hline \multicolumn{2}{|l|}{ About } & 3 \\
\hline \multicolumn{2}{|l|}{ What's EC? } & 3 \\
\hline \multicolumn{2}{|c|}{ Country-by-Country Information } & 15 \\
\hline \multicolumn{2}{|c|}{ EC Issues } & 9 \\
\hline \multirow{2}{*}{$\begin{array}{l}\text { Publications } \\
\text { and Resources }\end{array}$} & ICEC Publications & 23 \\
\hline & Partner Publications & 23 \\
\hline \multicolumn{2}{|l|}{ EC News } & 7 \\
\hline \multicolumn{2}{|l|}{ Total } & 83 \\
\hline
\end{tabular}

A primeira apresentação do processo de análise dos elementos que integram os argumentos contidos nos documentos está descrita no Quadro 3, a seguir.

Quadro 3: Principais argumentos para difusão dos CE por seção do website

\begin{tabular}{|c|c|}
\hline Seção do website & Principais argumentos para expandir $\mathrm{o}$ acesso à $\mathrm{CE}$ \\
\hline What's EC? & $\begin{array}{l}\text { - é importante porque se trata de um produto } \\
\text { seguro e eficaz para contracepção; } \\
\text { - é importante para reduzir riscos para a saúde das } \\
\text { mulheres; } \\
\text { - é um direito das mulheres como meio } \\
\text { indispensável à garantia de sua autonomia } \\
\text { reprodutiva. }\end{array}$ \\
\hline $\begin{array}{l}\text { Country-by-Country } \\
\text { information }\end{array}$ & $\begin{array}{l}\text { - redução de "gravidezes não desejadas"; } \\
\text { - CE é uma segunda chance para evitar "gravidez } \\
\text { não desejada"; } \\
\text { - o acesso é importante por meio de farmácias, sob } \\
\text { venda over the counter (OTC); } \\
\text { - altas taxas de fecundidade e "gravidez não } \\
\text { desejada"; } \\
\text { - altas taxas de morbidade e mortalidade maternas; } \\
\text { - altas taxas de abortamentos inseguros. }\end{array}$ \\
\hline
\end{tabular}




\begin{tabular}{|c|c|c|}
\hline \multicolumn{2}{|l|}{ EC Issues } & $\begin{array}{l}\text { - CE não é um medicamento abortivo (ênfase no } \\
\text { seu mecanismo de ação); } \\
\text { - CE deve ser vendida de forma OTC em farmácias; } \\
\text { - essencial em casos de violência sexual; } \\
\text { - essencial para adolescentes para as quais a } \\
\text { gravidez pode ser "questão de vida ou morte". }\end{array}$ \\
\hline \multirow[b]{2}{*}{$\begin{array}{l}\text { Publications } \\
\text { and } \\
\text { Resources }\end{array}$} & $\begin{array}{l}\text { ICEC } \\
\text { Publications }\end{array}$ & $\begin{array}{l}\text { - acesso importante porque se trata de um produto } \\
\text { seguro, eficaz e não abortivo (ênfase no mecanismo } \\
\text { de ação); } \\
\text { - reduzir o número de abortamentos e "gravidezes } \\
\text { não desejadas"; } \\
\text { - contraceptivo estratégico para garantia dos direitos } \\
\text { humanos e do imperativo da saúde pública; } \\
\text { - segunda chance para prevenção da gravidez; } \\
\text { - reduzir os riscos de "gravidez não desejada"; } \\
\text { - reduzir epidemia de "gravidez não desejada" e } \\
\text { abortamentos em "países em desenvolvimento". }\end{array}$ \\
\hline & $\begin{array}{l}\text { Partner } \\
\text { Publications }\end{array}$ & $\begin{array}{l}\text { - o acesso aos CE leva a benefícios de saúde, } \\
\text { sociais e econômicos para mulheres e sua família; } \\
\text { - redução de "gravidezes não planejadas", das taxas } \\
\text { de abortamentos e do número de crianças } \\
\text { negligenciadas e abandonadas; } \\
\text { - acesso importante porque se trata de um produto } \\
\text { seguro, eficaz e não abortivo (ênfase no mecanismo } \\
\text { de ação); } \\
\text { - direito de escolha, acesso é um direito humano; } \\
\text { - garantia de direitos reprodutivos; } \\
\text { - benefícios para saúde pública; } \\
\text { - aliviar o problema (social e econômico) da } \\
\text { gravidez na adolescência; } \\
\text { - gravidez na adolescência é um problema de saúde } \\
\text { pública; }\end{array}$ \\
\hline EC News & & $\begin{array}{l}\text { - redução de abortamentos e gravidezes "não } \\
\text { desejadas"; } \\
\text { - ponto de entrada para contracepção regular; } \\
\text { - é uma questão de saúde pública e direitos } \\
\text { humanos; } \\
\text { - direito das mulheres. }\end{array}$ \\
\hline
\end{tabular}

Esses resultados nos mostram que o ICEC possui três fortes linhas argumentativas cujos elementos se entrelaçam e se reforçam mutuamente: o argumento "sanitário"; o argumento 
"farmacêutico" e o argumento do "direito", os quais serão discutidos a seguir.

\section{O argumento sanitário $e$ as gravidezes "não-desejadas" como um problema de saúde pública}

Um dos argumentos mais difundidos para expandir o acesso aos CE é a questão das gravidezes imprevistas ou "não desejadas", como expressa nos documentos, $e$ as taxas de abortos provocados, como um "problema de saúde pública". De acordo com o ICEC, os contraceptivos de emergência poderiam reduzir a magnitude desse problema, por ser um reforço contraceptivo quando as mulheres "falham" em utilizar seus métodos regulares, reduzindo, assim, taxas de gravidezes "não desejadas". O argumento sanitário possui dimensões macro políticas, socioeconômicas e individuais, apontando que a gravidez "indesejada" gera problemas econômicos tanto para as mulheres, como para sua família e também para as nações.

Embora existam esforços do ICEC em defender que os $\mathrm{CE}$ seriam uma solução para esse problema, a coordenadora atual do consórcio, Elizabeth Westley, em editorial para a revista Contraception de 2012, relativiza tais expectativas. A autora afirma que as projeções/estimativas sobre o uso da CE para reduzir drasticamente o número de "gravidezes não planejadas" $e$, consequentemente a necessidade de serviços de aborto, não se confirmaram. Apesar de os $\mathrm{CE}$ reduzirem as chances de gravidez das mulheres, individualmente, em âmbito populacional as taxas de gravidez imprevistas não caíram. Para o consórcio, isso se deve ao fato de que as mulheres não estão utilizando os $\mathrm{CE}$, não os utilizam corretamente ou não possuem conhecimento da existência desses contraceptivos, o que ressalta a necessidade de novas mensagens de difusão no âmbito da saúde pública (Westley; Schwarz, 2012).

O grande foco na gravidez "indesejada" e no aborto como problemas sanitários permite refletir sobre uma preocupação que vem se constituindo no ocidente desde o século XIX. Trata-se, 
como Lupton (1995) designa, do imperativo da saúde que se configura como um conjunto de mecanismos pelos quais são assegurados a ordem, o crescimento canalizado de riquezas $e$ as condições de manutenção da saúde "em geral". A política médica delineada permite articular uma ética "privada", no caso uma ética "privada" das mulheres, para a boa saúde da população (Foucault, 2005; Lupton, 1995). As mulheres aparecem no material analisado como responsáveis por gerir a contracepção e alavancar uma "solução sanitária" para toda a população, reduzindo as "gravidezes não planejadas" $e$ as taxas de abortamentos ao se colocarem o tempo todo sob uma "vigilância contraceptiva". Ao "falharem" com seu método contraceptivo regular recomenda-se que procurem imediatamente o "back up control", o que aumenta as chances de os $\mathrm{CE}$ atingirem as expectativas populacionais $e$ sanitárias almejadas.

O diretor executivo da Family Planning Health Services escreveu em resposta a e-mails trocados no dia 13 de junho de 2012 sobre a provisão adiantada de CE (compra antecipada do contraceptivo antes da relação sexual), a qual seria, em sua opinião, a melhor forma de as mulheres utilizarem o contraceptivo, reduzindo assim as taxas de gravidez.

Eu estou convencido de que o Plan $B^{3}$ em provisão adiantada tem reduzido as taxas de gravidezes indesejadas de nossas pacientes como eu comuniquei (parcamente, tenho certeza) alguns anos atrás na conferência de Nova York. Nossas taxas de gravidezes em pacientes utilizando contraceptivos caiu de 5.5\%/ano para aproximadamente $2 \% /$ ano e tem se mantido assim pelos últimos 6 anos. Eu compreendo que as taxas de gravidezes entre mulheres na América do Norte não têm decaído, nem provavelmente em Wisconsin..., mas sob o princípio do "funciona se você

3 "Plan B" é uma expressão muito utilizada em língua inglesa, especialmente nos Estados Unidos da América, para se referenciar a contraceptivos de emergência. Notou-se o uso recorrente dessa expressão pelos membros do consórcio. 
usar", CE em provisão adiantada com explicação e lembretes, eu penso, funciona entre nossas clientes (Newman, L. grifo nosso, tradução nossa). ${ }^{4}$

Essas mensagens convidam a refletir sobre as pílulas contraceptivas poderem representar uma ampliação da autonomia reprodutiva feminina, em especial se considerarmos que, até o final do século XVIII e começo do século XIX, as mulheres dispunham de um arsenal limitado de técnicas para evitar uma gravidez. Mas é importante lembrar, como afirma Oudshoorn (2003), que no século XX nenhum novo método contraceptivo masculino foi desenvolvido, exceto o aperfeiçoamento dos já existentes, como o preservativo e a esterilização. Não obstante, vários novos métodos contraceptivos femininos foram criados, entre eles as pílulas hormonais, os dispositivos intrauterinos $e$ as pílulas hormonais de emergência. Além disso, de acordo com Cabral (2011), o fato de os homens poderem se abster de responsabilidades contraceptivas parece ter gerado maior responsabilização feminina.

Estudiosos como Berquó (1993) e Bozon (2005) também discutem que a difusão da contracepção pelo mercado e pela imposição de procedimentos médicos levou à queda das taxas de fecundidade no Brasil. Entretanto, isso não teve nenhum efeito sobre a redução da forte iniquidade de gênero no domínio da reprodução no país.

As imagens de mulheres com expressões faciais apreensivas ao lado de caixas de contraceptivos de emergência (Figura 2) e as imagens "objetivas", como os diversos gráficos utilizados pelo consórcio mostram que não é conferido à contracepção de

4 "I also am convinced that Plan $B$ in advance of need has reduced the unintended pregnancy rate of our patients as I communicated (poorly, I'm sure) a few years back at the $N Y$ meeting our pregnancy rate to contracepting patients dropped from 5.5\%/year to approx 2\%/year and has held there for the past 6 years. I understand the pregnancy rate among women in North America has not declined, nor probably even Wisconsin... but on the "it works if you take it" principle, EC in advance of need with explanation and reminders has, I think, worked among our clients" (Newman, L., grifo nosso). 
emergência o símbolo de medicamento que permitiria maior atividade sexual. Ao contrário, eles envolvem os sentidos sobre riscos à saúde, prevenção e uma "segunda chance" de proteção. Já a simbologia envolvida em propagandas sobre o Viagra ${ }^{\circledR}$ (Figura 3), por exemplo, privilegia bordões como "rigidez é o objetivo"; "rigidez mantém o equilibrio das relações"; "liberdade que permite ao paciente viver com maior espontaneidade e sem pressão de tempo" (Azize, 2002; Faro et alii, 2013).

Figura 2: Propaganda do Postpill ${ }^{\circledast}$ na Etiópia, retirada da página inicial do website do ICEC.

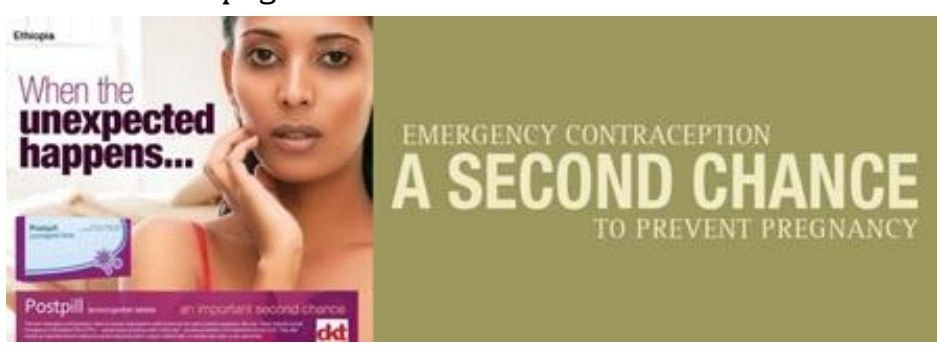

Figura 3: Propaganda da empresa Pfizer sobre Viagra ${ }^{\circledR}$.

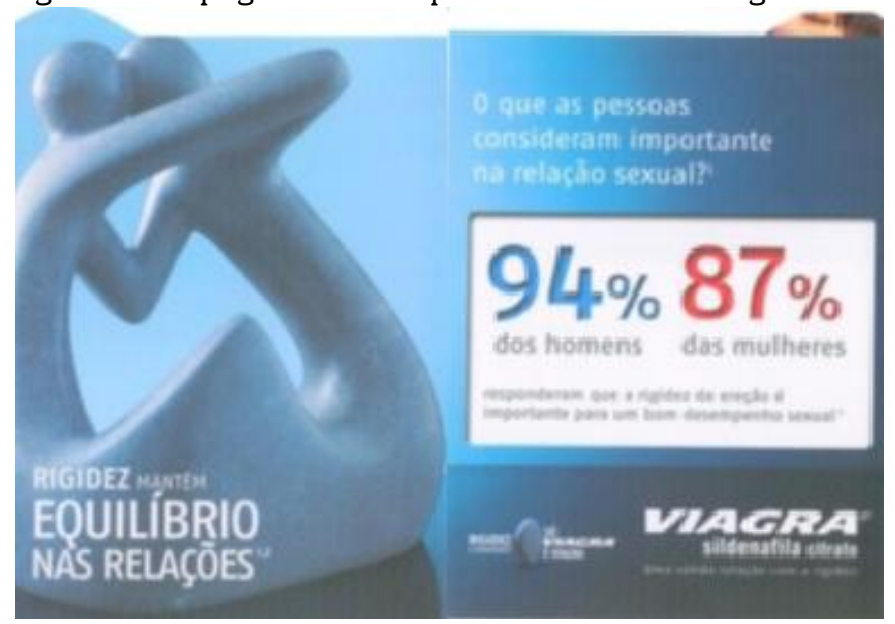

Fonte: Pfizer. In: Faro et alii (2013). 
No website do ICEC, não há destaque para o exercício da sexualidade feminina, ela se reduz à sua função reprodutiva. Ao invés de privilegiar a liberdade sexual que o $\mathrm{CE}$ pode conferir às mulheres, a preocupação sanitária revela uma "socialização das condutas de procriação" por meio de uma socialização médica, pelo valor patogênico atribuído às gravidezes (Foucault, 1977). O sexo enquanto campo de disputa política está o tempo todo permeando o argumento sanitário. $\mathrm{O}$ sexo se encontra, como demonstrou Foucault (1977), entre dois eixos que desenvolveram toda a tecnologia política da vida. De um lado, faz parte da disciplina do corpo - a disciplinarização dos corpos femininos para que utilizem os $\mathrm{CE}$. Do outro lado, integra a regulação das populações. Dessa forma, o bordão muito utilizado pelo ICEC para se referir à $\mathrm{CE}$, como uma "segunda chance" ou "última chance" para as mulheres "prevenirem" uma gravidez, é também uma "segunda chance" que concerne à dinâmica das taxas populacionais e melhoria da saúde pública.

\section{O argumento farmacêutico: o discurso sobre segurança, eficácia $e$ mecanismo de ação dos contraceptivos de emergência}

Outro argumento encontrado nos documentos veiculados pelo ICEC é o farmacêutico. Ele concentra a ampla divulgação de material biomédico enfatizando aspectos farmacológicos dos CE no website. Também está muito presente, apontando e dirimindo algumas inquietações em torno do contraceptivo. Segurança $e$ eficácia dos CE constituem pauta de diversas discussões e se apresentam como fortes premissas nos documentos, no sentido de que as mulheres devam acessá-los. Servem, igualmente, como suporte técnico na ampla campanha do consórcio para que os $\mathrm{CE}$ sejam distribuídos pelos farmacêuticos, profissionais que consideram estratégicos porque estão mais próximos do público em geral, ou mesmo que sejam distribuídos em farmácias de forma over-the-counter, ou seja, o cliente poderia acessá-lo sem qualquer interação com o profissional de saúde, diretamente nas gôndolas de produtos. 
Esse argumento se diferencia do argumento sanitário, primeiramente, por sua dimensão individualizante. Enquanto o argumento sanitário invoca a todo o momento a preocupação com a coletividade, o argumento farmacêutico envolve um discurso mais "exclusivo", dirigido à mulher. O ICEC associa a segurança e a eficácia como elementos fundamentais para a "obtenção" da "autonomia" feminina. Ao demonstrar essas características farmacológicas, o discurso ganha legitimidade científica para advogar em prol da "autonomia", do "empoderamento" feminino por meio dos CE. Mamo e Fosket (2009) sugerem que existe um discurso de marketing farmacêutico que tem associado os contraceptivos a liberdade, escolhas $e$ possibilidades, discurso que parece estar sendo incorporado pelo consórcio. A diferença é que, enquanto o argumento sanitário é normatizador, o argumento farmacêutico se detém em separar, estrategicamente, a política $e$ a ciência, no sentido de que o acesso à inovação científica e tecnológica beneficia a vida humana $e$, portanto, deve ser garantido, sem conotação moral ou política a priori.

Williams, Martin e Gabe (2011) refletem que os processos atuais escapam ao conceito de medicalização (Conrad, 2007), mas se encaixam na lógica da "farmacologização" (pharmaceuticalization), conceituada por eles como a tradução ou transformação de condições, capacidades e potencialidades humanas em oportunidades para intervenções farmacológicas. Ainda que com uma interface com a medicalização, a farmacologização não estaria necessariamente ligada a algum tipo de diagnóstico médico (Camargo, 2013). Por exemplo, a utilização de medicamentos "over-the-counter" (OTC), acesso que é amplamente defendido pelo ICEC em relação aos contraceptivos de emergência, demonstra a pertinência do conceito entre nós.

$\mathrm{O}$ acesso direto (OTC) aos contraceptivos de emergência é proposto como estratégico pelo ICEC, porque a farmácia seria o local de mais fácil acesso da população facilitando a obtenção dos $\mathrm{CE}$ em tempo oportuno. De acordo com o documento "Emergency Contraception: steps being taken to improve access", 
de 2002, publicado pela Guttmacher Institute e encontrado na subseção Partner Publications, há uma estimativa de que o fornecimento por meio desses estabelecimentos farmacêuticos preveniu 2000 "gravidezes não planejadas".

Esses discursos para ampliação do livre acesso (OTC) aos CE parecem servir tanto à ampliação da autonomia reprodutiva feminina quanto à intensificação dessa farmacologização - da sociedade e da sexualidade -, que se encontra imersa em uma biopolítica contemporânea. Os contraceptivos podem ser vistos como "tecnologias disciplinares" do comportamento de diversas maneiras. Mas ao mesmo tempo, dependendo do contexto em que se fala, os contraceptivos podem ser meios de libertação, oferecendo estratégias de resistência contra a disciplinarização de gênero, raça e classe (Clarke, 1998).

A campanha do ICEC para tornar a venda ou a distribuição dos CE isenta de mediadores, na forma OTC, é ampla no website e na lista de e-mails. $\mathrm{O}$ consórcio integra um projeto maior em parceria com o Reproductive Health Technologies Project (RHTP), que se intitula "ECOTC". O RHTP tem como principal objetivo: "que cada mulher possa atingir completa liberdade pelo acesso às tecnologias seguras, eficazes para que assegurem a si mesmas sua saúde e controle da sua fertilidade". A campanha em parceria com RHTP para o acesso OTC pode ser encontrada no website "http://rhtp.org/ECotc.asp" e contou com pedido de assinatura de petições e cartazes, como o visto abaixo, que dão um tom "jovial" à campanha (Figura 4). 
Figura 4: Cartaz de campanha pela venda OTC em farmácias.

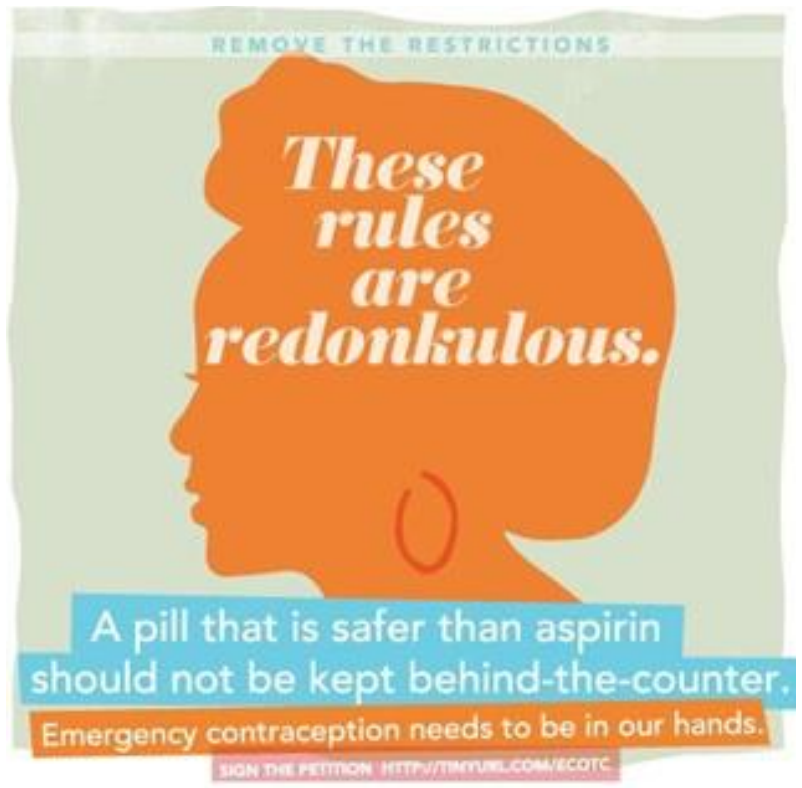

"Estas regras são ridículas. Uma pílula que é mais segura do que aspirina não deveria estar atrás do balcão. Contracepção de emergência precisa estar em nossas mãos" (tradução nossa). Fonte: Reproductive Health Technologies Project (RHTP), acessado em maio 2014: http://rhtp.org/ECotc.asp.

Essa argumentação pela venda OTC leva às últimas consequências o que Van Der Geest e Whyte (2011) argumentam quando dizem que os medicamentos são "libertadores", pois quebram a hegemonia dos profissionais $e$ habilitam as pessoas a se responsabilizarem por sua administração farmacológica. Os autores mostram estudos que demonstraram o distanciamento que os medicamentos podem agenciar entre usuários em relação aos especialistas. Por exemplo, os estudos de Allend Jr em 1970 demonstraram que as pessoas não procuravam tanto a ajuda profissional em hospitais, mas sim medicamentos. Esses locais eram vistos como fontes de medicação. Apesar de os 
medicamentos quebrarem a hierarquia com profissionais de saúde, um dos seus "encantos" é que, mesmo removidos de seu contexto, eles mantêm uma potencial conexão com médicos, laboratórios que os produziram, com a ciência biomédica que forma sua base essencial (Van Der Gesst; Whyte, 2011).

Além disso, eles podem também se tornar veículos de individualização, como no caso dos contraceptivos ou dos medicamentos para uso abortivo, pois as mulheres podem utilizálos de forma privada, diminuindo a dependência dos médicos e de outros profissionais. É a concretude dos contraceptivos de emergência que estabelece o seu "encanto", fácil de manusear após o ato sexual. São mercadorias que envolvem um acordo geral quanto ao seu valor e frequentemente visões divergentes de suas características e capacidades particulares (Van Der Geest; Whyte, 2011).

O mecanismo de ação (Mechanism of action - MOA) dos contraceptivos de emergência hormonais é um argumento central do ICEC para expansão do acesso aos CE. Dizer que eles não são abortivos é uma estratégia muito cara ao consórcio e se apresenta em praticamente todos os documentos analisados, pois consideram que isso tem surtido efeito positivo para expandirem os $\mathrm{CE}$, principalmente para países com cenários políticos conservadores, tendo ajudado a sua inserção em países onde o aborto é proibido, como no Brasil.

Para muitos setores conservadores da sociedade, os CE são abortivos (Souza; Brandão, 2012; Brandão et alii, 2017) porque se encontram em situação liminar: uma forma de contracepção utilizada após o coito. O consórcio se esforça em contraargumentar dizendo que eles não atuam após o óvulo fecundado já ter sido implantado e tenta "desmistificar" fatos, defendendo que os CE na sua forma farmacêutica de comprimido são "apenas uma pílula contraceptiva".

Em um documento produzido pela direção do ICEC, foram sintetizadas algumas discussões de como o ICEC aborda o MOA e as posições dos membros do consórcio, as quais podem ser distribuídas em quatro perspectivas. Em primeiro lugar, alguns 
membros concordaram que o ICEC deveria advogar por métodos pós-coito que agissem após a ovulação (como antiprogestinas em determinadas doses, por exemplo), ao invés de métodos que atuam na pré-fertilização - como o levonorgestrel. Isso porque consideram que os métodos pós-fertilização são mais efetivos, e para que não se fechem as portas para métodos que possam estar disponíveis no futuro. Consideram que focar apenas em métodos pré-fertilização poderia implicar que os métodos pós-fertilização não são aceitáveis pelo consórcio. Além disso, esses membros concordaram que o ICEC deveria se concentrar mais no porquê as mulheres precisam dos $\mathrm{CE}$, ao invés de focar no contraceptivo em si, em como ele age.

Em uma segunda perspectiva, membros do ICEC concordaram que o consórcio deve continuar promovendo os métodos atuais de contracepção de emergência, mesmo que não sejam tão efetivos quanto seriam se agissem após a ovulação. Para esses membros, não se deve enfatizar muito a falta de efetividade dos $\mathrm{CE}$, pois isso poderia se transformar em argumento para maior oposição em relação ao contraceptivo, limitando seu acesso. Uma terceira perspectiva dos interlocutores nessa troca de e-mails sobre o MOA se pautou em defender uma linha de argumentação que afastasse os CE do aborto. Para alguns, o fato de eles agirem apenas antes da ovulação pode ajudar a torná-los mais amplamente aceitos, particularmente entre aqueles que se opõem ao aborto. Por último, a direção do ICEC sintetizou que alguns integrantes se posicionaram pela perspectiva de que o consórcio deve modificar seu foco inteiramente voltado para o mecanismo de ação dos $\mathrm{CE}$ e contemplar mensagens de prevenção. Para esses, o ideal seria campanhas com slogans como: "It works if you use it" e "You can't have an abortion before you are pregnant". Além disso, acreditam que "a ciência não é atraente para todos" e que defender a expansão do acesso a esse contraceptivo apenas por meio de evidências científicas pode não atingir a todos. Para eles, as mensagens que não focassem no mecanismo de ação seriam mais efetivas para argumentar em defesa do acesso aos CE. 
Se, por um lado, alguns membros defendem menos ênfase sobre o contraceptivo em si e seu mecanismo de ação, por outro lado, alguns acreditam que o discurso sobre ele é estratégico para aumentar o acesso aos $\mathrm{CE}$, pois sua natureza científica seria "neutra" e capaz de purificar esse contraceptivo de todo tipo de abordagem política ou ideológica. É com esse argumento farmacêutico que o consórcio evidencia caminhos que discutem a "estabilização", isto é, um formato mais consensual e constante sobre os contraceptivos de emergência.

Quando os CE ainda não eram permitidos nos EUA para menores de 17 anos, Cleland et alii (2011), publicaram um comentário na revista Contraception em que diziam que a "política triunfa sobre a ciência". Argumentavam que não haveria motivos, a não ser políticos e ideológicos, para que esses contraceptivos não pudessem ser vendidos para todas as idades. $\mathrm{O}$ argumento farmacêutico é, portanto, um argumento chave para a expansão do acesso porque sustenta os $\mathrm{CE}$ enquanto fatos científicos "neutros".

De acordo com Clarke (1998), as interpretações que existem acerca das ciências reprodutivas, das quais os contraceptivos fazem parte, vão desde aquelas que enfatizam questões biológicas até as que consideram os dados científicos que respaldam os argumentos como uma blasfêmia. O que é moral, político, ideológico e científico está o tempo todo em debate quando se utiliza o argumento farmacêutico que enfatiza o mecanismo de ação dos CE. Como aponta a autora, dois tipos de política se cruzam nesse caminho: a política de fazer ciência se encontra com a política de modificar moralidades. O resultado disso é um trabalho científico que se atualiza apesar de seu status controverso.

Para parafrasear o antropólogo Victor Turner, os CE são medicamentos que estão num limiar, "betwixt and between", classificados como contraceptivos, mas usados depois do sexo. Essa característica liminar suscita o debate de diferentes formas em diferentes sociedades. Em países católicos e evangélicos, como os países da América Latina, o debate sobre CE é quase sempre centrado sobre o mecanismo de ação. Em contraste, no mundo 
muçulmano, o mecanismo de ação não tem sido um ponto importante, em parte pela diferença de interpretação do islã sobre o início da vida. O debate nesses países geralmente é centrado nas questões morais que a introdução dos $\mathrm{CE}$ acarretaria para as mulheres não casadas. Na Tunísia, esses contraceptivos foram introduzidos quase sem problemas, o que mostra que existe um contorno sociopolítico e também religioso sobre esse debate (Foster; Wynn, 2012).

Se por um lado temos, mais evidente no argumento sanitário, uma preocupação com a saúde da população, com o número de filhos e as questões demográficas e econômicas que uma gravidez inesperada pode acarretar, o argumento farmacêutico foca na responsabilidade individual pelo acesso privado aos contraceptivos de emergência, sendo responsabilidade da usuária se informar sobre segurança, eficácia, mecanismo de ação, posologia do contraceptivo, tendo também a possibilidade, caso deseje, de consultar um profissional de farmácia, seja ele o farmacêutico ou o balconista. Isso recorda o que Foucault (1990) chama de "tecnologias do eu". Para o autor, não se pode aceitar nenhum conhecimento como um valor dado, mas sim como "jogos de verdades" que se relacionam com técnicas específicas que as pessoas utilizam para entender a si mesmas. As "tecnologias do eu" permitem aos indivíduos efetuarem por conta própria, ou com a ajuda de outros, certo número de operações sobre o corpo, obtendo uma transformação ou atingindo um objetivo específico. A própria mulher deve equipar-se de um saber médico do qual pode servir-se permanentemente.

\section{$\mathrm{O}$ acesso aos contraceptivos de emergência como um direito}

O argumento que invoca o direito é, sobretudo, uma defesa ao direito da mulher aos contraceptivos como autonomia a ser garantida pela liberdade individual com responsabilidade coletiva, enfatizando o "empoderamento" feminino por meio dos CE. A defesa do direito aos contraceptivos como elemento central à 
saúde sexual e reprodutiva relacionado com o grau de liberdade individual e a própria responsabilidade com o coletivo não são ponderados nos argumentos dos materiais analisados nesta pesquisa. Questões sobre desigualdade entre os gêneros na esfera da sexualidade e da reprodução, ou sobre as vulnerabilidades femininas no âmbito da autonomia reprodutiva das mulheres são ausentes nos discursos. Não obstante, uma abordagem crítica nesse sentido nos parece central para compreensão dos limites $e$ possibilidades da liberdade $e$ autonomia feminina no acesso aos CE (Bastos; Ventura; Brandão, 2017).

Corrêa (2014) avalia que é nesse terreno que temos assistido perdas concretas, seja nos retrocessos de políticas públicas sobre o direito ao aborto, seja no esvaziamento do conteúdo dos direitos reprodutivos que não se referem à simples oferta de contracepção. O que vem ocorrendo atualmente é o que a autora chama de uma "política da oferta de contraceptivos", a qual vem promovendo o rápido retorno ao velho planejamento familiar que agora, como podemos perceber pelos documentos do ICEC, focaliza mais atentamente a África subsaariana e alguns países asiáticos.

$\mathrm{Na}$ sua análise crítica, Corrêa (2014) aponta que as organizações internacionais $e$ os Estados nacionais têm se comprometido apenas em implementar pautas mais "bemcomportadas" que emergiram da Conferência Internacional sobre População e Desenvolvimento (CIPD), ou seja, a saúde sexual e a saúde reprodutiva, deixando de lado ou resistindo mais abertamente às pautas mais radicais: gênero (na sua concepção mais complexa e instável), direitos reprodutivos e sexualidade. $\mathrm{O}$ conteúdo dos direitos que o ICEC defende é, principalmente, o direito ao acesso ao medicamento e à saúde reprodutiva, deixando subjacentes os direitos sexuais e reprodutivos, como podemos observar no excerto que segue.

Leis criminais e outras restrições legais que reduzem ou negam acesso a serviços e recursos de planejamento familiar, incluindo contracepção de emergência, violam o direito à saúde e refletem noções discriminatórias sobre o papel das mulheres na família e sociedade (Subseção "What 
do the experts say about EC?" contida em "What's EC?" website do ICEC, grifo nosso, tradução nossa). ${ }^{5}$

Segundo Corrêa e Ávila (2003), o direito à saúde reprodutiva é uma fusão entre direitos reprodutivos e saúde reprodutiva. Embora essa fusão seja muito habitual, segundo as autoras, é problemática. Isso pode levar a uma interpretação que dilua o significado do direito como prerrogativa de autonomia dos sujeitos nas esferas da sexualidade, das relações entre gêneros $e$ da reprodução. Por outro lado, esse vínculo entre direito e saúde possui ganhos inequívocos já que um sistema público universal e gratuito de saúde é um dos pilares inegociáveis para a promoção da igualdade e do exercício pleno dos direitos sexuais $e$ reprodutivos. Entretanto, dizer que esse sistema universal $e$ gratuito de saúde é crucial para a redução da desigualdade social e racial em saúde não resolve fatores que restringem os direitos reprodutivos e sexuais das mulheres, aqueles que estão no âmbito das relações interpessoais de gênero, por exemplo.

Para dar fôlego ao argumento do direito ao acesso ao medicamento, o ICEC enfatiza sua inclusão nas Listas de Medicamentos Essenciais (LME). Essa ênfase nas LME é estratégica porque elas são instrumentos importantes para o planejamento da Assistência Farmacêutica no mundo e expressaria uma forma de garantia do direito à saúde que inclui o acesso a medicamentos. Um medicamento incluído nessas listas passa a ser constantemente adquirido e disponibilizado pelos países e logo se formaliza como um direito para os cidadãos e cidadãs (Hunt, 2008).

De acordo com o documento de 2003 (Emergency Contraception in National Essential Medicines Lists), contido na ICEC Publications, a lista de medicamentos essenciais (LME) da

5 "Criminal laws and other legal restrictions that reduce or deny access to family planning goods and services, including emergency contraception, violate the right to health and reflect discriminatory notions of women's roles in the family and society (Subseção "What do the experts say about EC?" contida em "What's EC?" website do ICEC, grifo nosso). 
Organização Mundial da Saúde (OMS) (março de 2011) inclui o levonorgestrel. Inclui tanto a apresentação com os dois comprimidos de $0,75 \mathrm{mg}$ quanto com 1 comprimido de $1,5 \mathrm{mg}$. Segundo o documento, dos 113 países que possuem uma LME disponível, 58 contêm as pílulas contraceptivas de emergência. ${ }^{6} \mathrm{O}$ Brasil apresenta, desde 2010, o CE - no regime de levonorgestrel $1,5 \mathrm{mg}$ - em sua LME divulgada pela Agência Nacional de Vigilância Sanitária (ANVISA). Para a OMS, os medicamentos essenciais se constituem como principais instrumentos para a realização de uma efetiva política de medicamentos.

Biehl (2007), Greene (2010), Biehl e Petryna (2011) são autores que refletiram sobre o direito à saúde estar se transformando no direito ao acesso a medicamentos. Biehl (2007), ao observar o contexto do HIV/AIDS no Brasil, discute que diversas ONGs, em parceria com o Banco Mundial, emolduraram uma demanda por acesso aos antirretrovirais enquanto um direito humano, de acordo com a Constituição Federal que prevê o direito à saúde (Dallari, 2009), e ativistas realizaram o lobby por uma legislação específica para tornar os medicamentos universalmente disponíveis.

De acordo com Greene (2010), é crescente a centralidade dos medicamentos em discussões e propostas de intervenção de organizações internacionais como a OMS e entidades filantrópicas como a Bill and Melinda Gates Foundation, na área da saúde, inclusive em questões de saúde reprodutiva com parceria dessa fundação com o ICEC. Segundo o autor, nove dos quatorze

${ }^{6}$ De acordo com a nova Lista de Medicamentos Essenciais (LME) da OMS (WHO, 2017), publicada em 6 de junho de 2017, além das apresentações de levonorgestrel com os dois comprimidos de $0,75 \mathrm{mg}$ e 1 comprimido de $1,5 \mathrm{mg}$, o Ulipristal Acetato (UPA) 30mg também agora é considerado essencial, sendo considerado como mais eficaz do que o levonorgestrel, pois estudos clínicos randomizados demonstram que UPA preveniu mais gravidezes do que quando comparado ao levonorgestrel (Denielsson, Gemzell e Cameron, 2011). O Brasil incorporou, em 2010, CE no regime de levonorgestrel $1,5 \mathrm{mg}$ e $0,75 \mathrm{mg}$ na sua LME, divulgada pela ANVISA e atualizada em 2014. No Brasil, o Ulipristal ainda não é considerado essencial $e$ foi recentemente registrado pelo órgão regulador de medicamentos, ANVISA (WHO, 2017). 
desafios lançados no Global Challenge ${ }^{7}$, da Fundação Bill and Melinda Gates, se referem ao fornecimento de medicamentos. O objetivo desse projeto é incentivar iniciativas científicas $e$ tecnológicas que resolvam os "problemas-chaves" de saúde nos "países em desenvolvimento".

Há meio século, a centralidade dos medicamentos não era tão óbvia. A constituição de 1946 da OMS não os mencionava. Quando a OMS publicou, em 1977, uma lista com 186 "medicamentos essenciais" definidos como "básicos, indispensáveis e necessários para a saúde das populações", tornou os medicamentos como questões-chave na política econômica internacional (Grenne, 2010). Essa "essencialidade" sustenta as estratégias de grupos de advocacy para descrever a falta de acesso aos medicamentos como algo moralmente inaceitável. Organizações não governamentais cada vez mais têm contornado a soberania de países e detentores de patentes em seus esforços de trazer os medicamentos essenciais para populações necessitadas de condições básicas de sobrevivência.

Assim, essa discussão vem se dando em paralelo ao debate dos direitos humanos. Ainda, segundo Greene (2010), o lobby de laboratórios farmacêuticos dentro da OMS contribuiu para que listas de medicamentos essenciais deixassem de lado medicamentos novos, com alto potencial de carrear lucros, fazendo com que fossem essenciais os medicamentos há mais tempo no mercado. $\mathrm{O}$ direito à saúde está sempre mais afetado pelas novas configurações do biopoder, cujas intervenções se manifestam não só pelas ações do Estado, mas pelo poder de organizações internacionais e do mercado de tecnologias de saúde. As empresas biotecnológicas suscitam crescentes demandas de consumo de saúde, buscando configurar o seu consumo como um direito, sem a devida e adequada hermenêutica do direito ao acesso aos medicamentos e outras tecnologias com o direito à

${ }^{7}$ Essa iniciativa da Fundação Bill e Melinda Gates pode ser acessada em Grand Challenges in Global Health pelo endereço: http://www.grandchallenges.org/Pages/Default.aspx. 
saúde e os direitos humanos, especialmente, em relação às desigualdades e iniquidades presentes nessa configuração (Junges, 2009).

Ao se apropriar dessa linguagem dos direitos, o consórcio reconhece, sobretudo, o direito ao acesso a contraceptivos, deixando subordinado a este direito os direitos sexuais $e$ reprodutivos. Ele cria subjetividades, moralidades e também um mercado consumidor. Dessa forma, um consórcio inteiramente dedicado aos contraceptivos de emergência, que advoga pelo direito das mulheres ao acesso aos $\mathrm{CE}$, traduz direitos sexuais $e$ reprodutivos no direito de consumidoras acessarem tais medicamentos.

Essas organizações que constituem o ICEC tiveram e ainda têm um papel importante na difusão de contraceptivos ao redor do mundo e também no Brasil. Os argumentos que utilizam para essa expansão foram e continuam sendo fundamentais nas políticas de planejamento reprodutivo e afetam também as experiências, sobretudo das mulheres, com essas tecnologias. $\mathrm{O}$ que fica claro nos documentos analisados é que nossos corpos, especialmente os femininos, estão imersos em um campo de responsabilidades e escolhas. A partir dos argumentos analisados, temos uma boa demonstração da biopolítica contemporânea (Rose, 2013) - uma forma de poder coletivizador e ao mesmo tempo individualizador que busca expressar uma preocupação com o bem estar de "todos".

\section{Considerações finais}

Esta pesquisa teve por objetivo compreender os sentidos subjacentes aos argumentos do Consórcio Internacional sobre Contracepção de Emergência. É possível dizer que a linguagem biomédica está muito bem documentada e se configura um enunciado importante para justificar a necessidade do acesso aos contraceptivos de emergência pelas mulheres. $\mathrm{O}$ ativismo que pretende torná-los acessíveis a todas as mulheres se ocupa fortemente com um discurso farmacológico, no qual a centralidade 
do princípio ativo é notável na defesa de que um componente químico e sua segurança seja garantia de escolhas e uma segunda chance de empoderamento e liberdade para mulheres.

Os argumentos sanitários se ancoram na promessa de que, ao expandir o acesso aos $\mathrm{CE}$, as taxas de abortamentos $e$ gravidezes imprevistas se reduziriam. Nesse sentido, demonstra que uma questão importante para o ICEC é reforçar o acesso aos $\mathrm{CE} e$ os efeitos da pílula no âmbito populacional. O discurso centrado na saúde pública para redução de taxas de gravidez imprevista e de abortamentos é central nesses argumentos.

$\mathrm{O}$ discurso farmacêutico se fortalece no sanitário. A afirmação de Wynn (2012) é exemplar na medida que reforça a ideia de que se as mulheres tivessem uma "última chance" de prevenção da gravidez haveria redução de "gravidezes indesejadas" e de abortamentos inseguros. O acesso sem a prescrição seria importante para a redução da morbidade relacionada à gravidez e de custos para serviços de saúde. Dessa forma, diante do "fracasso" de defender o acesso sem prescrição como medida de saúde pública, Wynn (2012) diz que nas reuniões do ICEC discutiu-se mudar o foco da argumentação da saúde pública para os direitos individuais. Tal deslocamento acaba tornando o medicamento um dispositivo que mescla segurança, prevenção e autonomia.

Essa articulação de uma ordem sanitária e uma ordem farmacológica para o direito individual ao acesso aos contraceptivos de emergência que protegem as mulheres de uma "gravidez indesejada", de complicações que podem decorrer de uma gravidez, faz pensar que não há um "descolamento" da sexualidade de um contexto potencial de perigo. Em outras palavras, a sexualidade feminina é abordada na perspectiva do risco e não do direito ao livre exercício da sexualidade. A sexualidade feminina aparece no website como um objeto socialmente útil e politicamente conservador. A ausência do debate sobre as desigualdades de gênero que pautam o exercício da sexualidade heterossexual e a prática da violência sexual masculina são lacunas importantes não contempladas. A 
farmacologização do corpo da mulher é um dispositivo que relaciona questões políticas mais gerais, relativas à população, $e$ aspectos mais individuais de cuidado com o corpo feminino (Vieira, 2003).

Compreender os argumentos para a difusão dos contraceptivos de emergência pelo ICEC proporcionou refletir sobre os discursos sobre esses novos métodos, na percepção desse consórcio, e assumir que eles são soluções científicas de alto nível tecnológico e farmacológico que podem garantir ampliações da autonomia feminina e alavancar alternativa sanitária para responder a taxas de aborto e gravidezes imprevistas. Ao mesmo tempo que é possível constatar que a mensagem subjacente de que as mulheres só encontram as "liberdades" e "escolhas" possuindo acesso aos contraceptivos de emergência pode reduzir o alcance da concepção dos direitos sexuais e reprodutivos. Dessa forma, consideramos que este trabalho contribui para elucidar as bases sob as quais o ativismo do ICEC se sustenta, demonstrando que velhas e novas argumentações se fazem presentes entre nós, especialmente no que tange aos corpos femininos.

\section{Referências bibliográficas}

AGÊNCIA Nacional de Vigilância Sanitária. O que devemos saber sobre medicamentos? Brasília, DF, ANVISA, 2010.

AzizE, Rogério Lopes. A química da qualidade de vida: um olhar antropológico sobre o uso de medicamentos e saúde em classes médias urbanas brasileiras. Dissertação (Mestrado em Antropologia Social) - Universidade Federal de Santa Catarina, Florianópolis, 2002.

BAStos, Luiza Lena; VentuRA, Miriam; BRANDÃO, Elaine Reis. Saúde sexual e reprodutiva, conservadorismo religioso $e$ acesso a medicamentos: uma discussão sobre a estratégia global de advocacy do Consórcio Internacional sobre Contracepção de Emergência. Sex., Salud Soc. n²6, Rio de Janeiro, 2017, pp.306-327. 
BERQUÓ, Elza. Brasil, um caso exemplar: a anticoncepção e partos cirúrgicos à espera de uma ação exemplar. Revista Estudos Feministas vol. 1, nº 2, 1993, pp.367-381.

BIEHL, João. Pharmaceuticalization: AIDS treatment and Global Health Politics. Anthropological Quarterly vol. 80, n 4, 2007, pp.1083-1126.

; Petryna, Adriana. Bodies of rights and therapeutic markets. Social research vol. 78, n 2, 2011, pp.359-386.

BONAN, Claudia. Reflexividade, sexualidade e reprodução: encruzilhadas da modernidade latino-americana. Iberoamericana vol.18, 2005, pp.89-107.

Bozon, Michel. L'évolution des scenarios de la vie reproductive des femmes au Brésil. Médicalisation, genre et ingalités sociales. TiersMonde vol. 46, $\mathrm{n}^{\circ}$ 182, 2005, pp.359-384.

BRANDÃO, Elaine Reis et alii. Os perigos subsumidos na contracepção de emergência: moralidades e saberes em jogo. Horizontes Antropológicos vol. 23, n 47, Porto Alegre, 2017, pp.131-161.

CABRAL, Cristiane Silva. Práticas contraceptivas e gestão da heterossexualidade: agência individual, contextos relacionais $e$ gênero. Tese (Doutorado em Saúde Coletiva) - Instituto de Medicina Social, Universidade do Estado do Rio de Janeiro, Rio de Janeiro, 2011.

CAMARGO, Kenneth Rochel. Medicalization, pharmaceuticalization and health imperialism. Cadernos de Saúde Pública vol. 29, n 5, Rio de Janeiro, 2013, pp.844-846.

ClARKE, Adele. Disciplining reproduction: modernity, american life sciences, and "the problem of sex". Berkeley, University of California Press, 1998.

ClelAND, Kelly et alii. Plan B, one step not taken: politics trumps science yet again. Contraception vol. 85, n 4, 2011, pp.340-341.

CONRAD, Peter. The medicalization of society: on the transformation of human conditions into treatable disorders. Baltimore, The John Hopkins University Press, 2007.

CORRÊA, Sônia. Legados do Cairo: para além da "mesmice"? In: WONG, L.R. (org.). Cairo +20: perspectivas da agenda de população e 
desenvolvimento sustentável pós 2014. Rio de Janeiro, ALAP, 2014. pp.23-31.

; AviLA, Maria Betânia. Direitos sexuais e reprodutivos: pauta global e percursos brasileiros. In: BERQUÓ, E. (org.). Sexo \& vida: panorama da saúde reprodutiva no Brasil. Campinas, SP, Ed. Unicamp, 2003, pp.17-78.

DALLARI, Sueli. O conteúdo do direito à saúde. In: CoSTA, Alexandre Bernardino et alii (org.). $O$ direito achado na rua: introdução crítica ao direito à saúde. Brasília, DF, CEAD/ UNB, 2009, pp.91-101.

DENIELSSON, Kristina Gemzell; CAMERON T. Sharon. Ulipristal acetate (ellaOne ${ }^{\circledR)}$ for emergency contraception review of the clinical evidence. Clin. Invest, vol.1, n³, 2011, pp.467-472.

DesclauX, A.; LÉVY, J.-J. Présentation: culture et médicaments. Ancien objet ou nouveau courant en anthropologie médicale? Anthropologie et Sociétés 27(2), 5e21, 2003.

ELLERTSON, Charlotte. History and efficacy of emergency contraception: beyond Coca-Cola. Family Planning Perspectives vol. 28, n² 2, 1996, pp.44-48.

FARO, Livi et alii. Homem com "H". Ideais de masculinidade (re)construídos no marketing farmacêutico. cadernos pagu (40), Campinas, SP, Núcleo de Estudos de Gênero-Pagu. Unicamp, 2013, pp.287-321.

FOSTER, Angel M.; WYNN, L. L. (org.) Emergency contraception: the story of a global reproductive health technology. New York, Palgrave Macmillan, 2012.

FouCAult, Michel. História da sexualidade I: a vontade de saber. Rio de Janeiro, Graal, 1977.

- Tecnologías del yo $Y$ otros textos afines. Barcelona, Paidós Iberica, 1990, pp.45-94.

- A política da saúde no século XVIII. In:

Microfísica do poder. 21ed. Rio de Janeiro, Graal, 2005, pp.193-207.

GREENE, Jeremy. A. When did medicines become essential? Bulletin of World Health Organ vol. 88, n 7, 2010, pp.483-484. 
HASPELS, A. A.; ANDRIESSE, R. The effect of large doses of estrogens post coitum in 2000 women. European Journal of Obstetrics \& Ginecology and Reproductive Biology vol. 3, n 4, 1973, pp.113-117.

HunT, Paul; KhOSLA, Rajat. Acesso a medicamentos como um direito humano. Sur. Revista de Direitos Humanos vol. 5, n 8, 2008, pp.99115.

JUNGES, José Roque. Direito à saúde, biopoder e bioética. Interface Comunicação, Saúde, Educação vol. 13, n² 29, 2009, pp.285-295.

LUPTON, Deborah. The imperative of health: public health and the regulated body. London, Sage, 1995.

MAMO, Laura; FOSKET, Jennifer. R. Scripting the body: pharmaceuticals and the (re)making of menstruation. Journal of Women in Culture and Society vol. 34, n 4, 2009, pp.925-949.

MANICA, Daniela. Contracepção, natureza e cultura: embates e sentidos na etnografia de uma trajetória. Tese (Doutorado em Antropologia Social) - Universidade Estadual de Campinas, Campinas, 2009.

OUDSHOORN, Nelly. Beyond the natural body: an archeology of sex hormones. New York, Routledge, 1994.

. The male pill: a biography of a technology in the making. Durham, Duke University Press, 2003.

PRESCOTT, Heather Munro. The morning after: a history of emergency contraception in the United States. Ney Jersey, Rutgers University Press, 2011.

ROSE, Nikolas. A política da própria vida: biomedicina, poder $e$ subjetividade no século XXI. São Paulo, Paulus, 2013.

SoUZA, Rozana Aparecida; BRANDÃO, Elaine Reis. À sombra do aborto: o debate social sobre a anticoncepção de emergência na mídia impressa brasileira (2005-2009). Interface vol. 16, $\mathrm{n}^{\circ}$ 40, Botucatu, 2012, pp.161-175.

TEIXEIRA, M. et alii. Representations and uses of emergency contraception in West Africa. A social anthropological reading of a northern medicinal product. Social Science \& Medicine vol. 75, $\mathrm{n}^{\circ}$ 1, 2012, pp.148-155. 
TRUSSELL, James. Emergency contraception: hopes and realities. In: FOSTER, Angel. M.; WYNN, L. L. (org.). Emergency contraception: the story of a global reproductive health technology. New York, Palgrave Macmillan, 2012, pp.19-35.

VAN Der Geest, Sjaak; WhYTE, Susan Reynolds. O encanto dos medicamentos: metáforas e metonímias. Sociedade e Cultura vol. 14, $\mathrm{n}^{\circ} 2,2011, \mathrm{pp} .457-472$.

VIEIRA, Elisabeth Meloni. Políticas públicas e contracepção no Brasil. In: BERQUÓ, Elza. Sexo \& vida: panorama da saúde reprodutiva no Brasil. São Paulo, Ed. Unicamp, 2003, pp.151-196.

WeSTLEY, Elizabeth; ScHWARZ, E.B. Emergency contraception: global challenges, new opportunities. Contraception vol. 85, 2012, pp.429431.

Williams, Simon J; MARTIN, Paul; GABE, Jonathan. The pharmaceuticalisation of society? A framework for analysis. Sociology of Health \& Ilness vol. 33, n 5, 2011, pp.1-16.

WORLD Health Organization. Consensus statement on emergency contraception. Contraception n ${ }^{\circ}$ 52, 1995, pp.211-213.

WYNN, L.L. United States: Activism, sexual archetypes, and the politicization of science. In: FOSTER, A. M.; WYNN, L. L. (org.). Emergency Contraception: the story of a global reproductive health technology. New York, Palgrave Macmillan, 2012, pp.39-55.

\section{Fontes}

INTERNATIONAL Consortium for Emergency Contraception [http://www.cecinfo.org - acesso de mar. 2013 a julho 2014].

REPRODUCTIVE Health Technologies Project (RHTP) [http://rhtp.org/ acesso em maio 2014].

THE BILL and Melinda Gates Foundation. Grand Challenges in Global Health [https://goo.gl/tjuCu3 - acesso em maio 2014].

U.S. Food and Drug Administration (FDA): Understanding over-thecounter medicines [https://goo.gl/1RASJY - acesso em set.2017].

WORLD Health Organization. Model List of Essential Medicines, 20th List, 2017 [goo.gl/Q3RAxg - acesso em set. 2017]. 